\title{
Research on the Visualization of Nanyin Characteristic Resources Based on Topic Maps
}

\author{
Jiantao Liu, a, *, Ruiming Fang ${ }^{2, \text { b }}$ \\ ${ }^{1}$ Library of Huaqiao University, Quanzhou 362021, China \\ ${ }^{2}$ Department of Electrical Engineering, Huaqiao University, Xiamen 361021, China

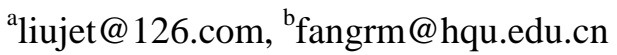

Keywords: Characteristic digital resources, visualization, topic maps, OKS, Nanyin.

\begin{abstract}
The main method users utilize digital resources is through classification and retrieval functions. However, it is not ideal for the use of some specialized characteristic resources. Taking Nanyin (an intangible music culture heritage from China) as an example, we analyze the different topic objects and semantic association of Nanyin characteristic resources by using topic maps theory. In addition, we apply OKS to describe and reveal them, and present a complete Nanyin knowledge network using visualization. This research of knowledge visualization can increase digital resources utilization and improve user experience.
\end{abstract}

\section{Introduction}

In the Era of Information explosion, the main focus of digital resource construction has shifted from maximizing resource quantity to meeting the users' diversified and multi-level knowledge needs. Therefore, effectively revealing the knowledge objects and their relations contained in digital resources has become a necessary requirement for resource construction to adapt to the knowledge-based information service. Traditional digital resources mostly provide classified navigation and retrieval functions, but the correlation between information is weak and the distribution is relatively scattered. Topic map is a visual knowledge organization method. It is independent of the technology platform and can construct structured semantic relations on multiple information resource platforms.

The main applied studies on resource organization related to topic map technology include the following. R. Schweiger uses topic map tools to correlate medical clinical data, allowing linking of various web-based data [1]; Qingmao Li uses it to organize tourism cultural information [2]; Yingfan Gao uses it to restore the data from the missing Malaysia Airlines Flight MH370 [3]; Hui Qu proposes a multidimensional semantic indexing method that based on the metadata of books and journals [4]. However, little research has been done on the characteristic digital resources, especially the resources on highly specialized intangible cultural heritage. Taking Nanyin characteristic resources as an example, this paper intends to make a topic map and reveal Nanyin knowledge network with visualization technology. Our research helps users acquire accurate Nanyin knowledge and promote the inheritance of intangible culture.

\section{Nanyin Characteristic Resources}

Characteristic resources collect information on specific subjects, topics and people, which are classified, sorted, stored during a specific historical period, or at a specific geographical area. For instance, Nanyin, the world's intangible cultural heritage, is the oldest existing traditional ancient music in China and is called the living fossil in the history of Chinese music. Nanyin has 3 unique characteristics.

Highly professional: The music score has a unique way of recording; the instrument has the ancient proper nouns; performer sing in South Fujian dialect; users need to possess basic knowledge 
of Nanyin in order to use the resources.

Complex inheritance relationship: Nanyin originated from more than a thousand year ago, it is difficult to explore the history of skill inheritance and genres evolution.

Scattered and diversified resources: Nanyin culture spreads all over the Southeast Asian countries and regions through Nanyin communities. Various kinds of books, research papers and video resources are also scattered.

Therefore, if users do not possess basic knowledge of Nanyin, they will find it difficult to utilize Nanyin resources, or they won’t even know what keywords to use for searching.

\section{Nanyin Topic Map Model}

We establish a Nanyin topic map model according to the definition of topic maps in international standard ISO/IEC13250 [5]. As seen on figure 1, the model consists of 2 layers. The top layer is knowledge layer that has nodes indicating different topic types. The types of topics can include anything that users would like to discuss about. The lines connecting the nodes represent associations, describing the semantic relations between topics. The bottom layer is occurrence layer, displaying all the information resources related to the topic. A topic can be guided by dashed lines to one or more of its associated information resources, forming the association between the upper and lower layers.

The independent relationship between the upper and lower layers has two advantages: Firstly, the knowledge layer can cover multiple information resources, effectively solving the problem of insufficient and scattered single Nanyin resources; secondly, multiple sets of information resources can be constructed on the same information resource topic map, supplying readers with personalized viewing perspective.

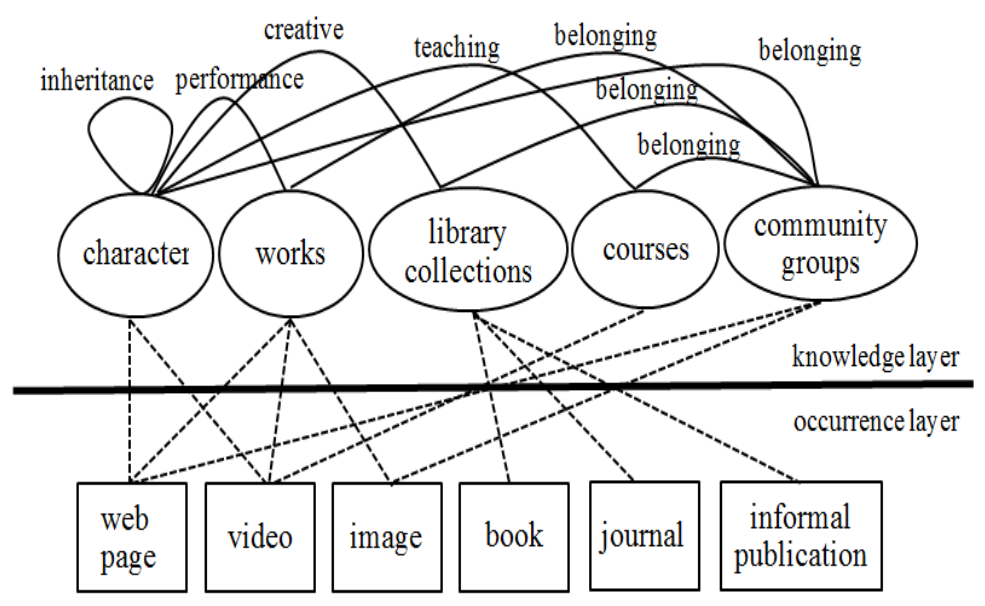

Fig. 1 Nanyin Topic Map Model

\section{Core Elements of the Model}

\subsection{Topic Type Analysis}

Topic type, association and occurrence are the core of constructing a topic map. In figure 1, we use hierarchy to show topic types, and each top-level topic has their sub types.

Character types: Nanyi's skills and knowledge are demonstrated by the teachers and passed down to next generation verbally. There are 3 main sub types: Inheritors, Nanyi masters and scholars. Inheritors are the designated heir of Nanyin. Nanyi Masters refer to the well-known Nanyin performers and Song writers. Scholars are researchers who write the relevant documents of Nanyin.

Works types: Nanyin works are colorful and versatile. They are divided into 3 types: Finger, music scores and songs. Finger works refer to the set of Nanyin pieces that have lyrics, music scores and also specific instructions for traditional Chinese folk instrument PiPa playing including fingering. 
Their content is usually derived from opera, folk art stories and legends. Score works are the set of Nanyin pieces that have music scores, specific instructions for PiPa playing, but no lyrics. Their content usually includes 4-season scenery, flowers, birds and insects, or racing horses. Songs refer to the more than 6000 standalone Nanyin pieces, which account for the largest number of Nanyin works.

Library collections: Include 3 types: books, research papers, and informal publications. Libraries collect Nanyin related documents including formal published and distributed books, research papers, those historically preserved songs, lyrics, classical stories, ancient poetry, couplets and etc.

Community groups: Community groups are important institutions that conduct Nanyin academic research, and organize performance activities disseminate Nanyin culture. Community groups are divided into 4 sub-groups by different functions and geographical areas: study institutes, college orchestras, domestic communities, and overseas communities.

Course types: Course materials include online videos of Nanyin Instruction. The videos are divided into 4 sub-groups: singing technique instruction, musical instrument instruction, Nanyin notation and interviews of Nanyin masters.

\subsection{Association Types Analysis}

Association describes the relationship between any topic. Association relationship can be one to many, or many to many, eventually forming a complex network of associations. In the Nanyin theme, we define 5 kinds of associations.

Inheritance relationship: When it comes to non-material cultural heritage, mentoring relationship is the most basic and formal inheritance relationship. Nevertheless, with the increasing popularity of Nanyin, professional teachers or scholars also partly play a role of spreading Nanyin culture. Hence, the relationship between Chuan (the role of passing down Nanyin knowledge) and Cheng (the roll of inheriting) has been expanded. Non-hereditary can be inherited from the Nanyin characters under the broad categories.

Performance relationship: Nanyin works generally require the participation of singers and performers. Performance relationship specified in the Nanyin works consist of characters, such as masters, inheritors and so on.

Creative relationship: Nanyin books, research papers, reports, lyrics and music are often written by Nanyin scholars or masters. The creative relationship indicates the relationship between the work and the author.

Teaching relationship: With the continuous enrichment of community activities and online classes, the teaching relationship defines relationship between instructional videos and teachers. It reveals the direct or indirect connection of online courses.

Belonging relationship: The smallest Nanyin performance unit is often the community group. Defining the belongings of performers and community groups better sort out the development of various associations and the evolution of different genres.

\subsection{Resource Occurrence Types}

Top map uses resource occurrence to guide users to relevant information about a particular topic. In this paper, the resource types are mainly digital documents such as images, videos, books, journals, or related text descriptions from "Quanzhou Nanyin Characteristic Resource Library" and "Fujian Nanyin Network".

\section{Topic Map Generation and Visualization}

Commonly used topic maps generation tools are TM4J, tinyTIM, XTM4XMLDB and OKS. OKS (Ontopia Knowledge Suite) is a Java based open source software, currently the most complete and widely used open source topic tool [6]. Our research uses OKS to create, maintain and configure Nanyin topic map.

The OKS structure consists of the engine interface, storage layer and the OKS sample tools. The engine is responsible for the data exchange and core operation of modifying, accessing, storing the 
topic maps. Storage layer stores user input data in RAM by default. Here we use MySQL database to prevent data loss caused by power outage. The application layer provides many user interfaces, e.g. Ontopoly, which can edit ontology and instance instances and output the data in various formats such as XTM and RDF. Omnigator and Vizigator can browse and visualize the theme respectively.

Nanyin topic navigation map is shown in Figure 2. This is built based on the definition of the Nanyin topic and the web application interface provided by OKS. The graph shows not only all inheritors of Intangible Cultural Heritage, but also their performances, their various types of works, and collaborative performances. Clicking on each topic expands subnodes or directly accesses associated digital resources. Key words can also highlight the topic of retrieval, to achieve rapid positioning.

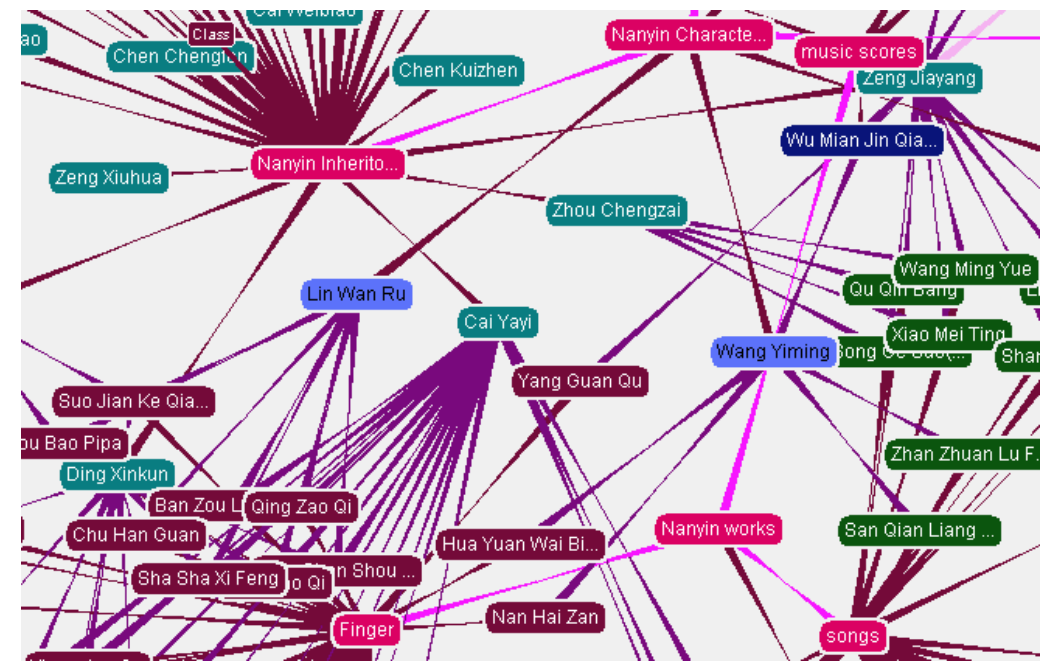

Fig. 2 Part of Nanyin Topic Map

\section{Conclusions}

Based on the topic maps theory and method, this paper not only systematically sorts out the structure of the Nanyin related knowledge, but also provides visual browsing and retrieval through the definition and analysis of the Nanyin Topic, association and resource indexes. This remedies the shortcomings of traditional information organization methods, and significantly improves the protection and inheritance of intangible cultural. At the same time, this paper provides meaningful reference to the construction of other similar characteristic resources.

\section{Acknowledgments}

The research is supported by the education research project for middle-aged and young teachers of Fujian, China (No. JZ170338).

\section{References}

[1] Schweiger R, Hoelzer S, Rudolf D, Rieger J, Dudeck J. Linking clinical data using XML topic maps. Artificial Intelligence in Medicine. 2003, 28(1): 105-115.

[2] Qingmao Li. Research on topic maps based tourism document organization method. Data Analysis and Knowledge Discovery. 2009(4): 82-87.

[3] Yingfan Gao, Ying Li, Lingen Meng. Application study of topic maps in emergency information analysis. Information Stuyies: Theory \& Application. 2016(6): 115-119.

[4] Hui Qu, Lei Zhou. Research on multidimensional semantic indexing of digital resource of collection based on topic relevance. Library Development. 2016(4): 47-51, 57. 
[5] Smits G F, Jordaan E M. Improved SVM regression using mixtures of kernels. Proceedings of the 2002 International Joint Conference on neural networks. Hawaii, 2002: 2785-2790.

[6] Yejun Wu, David J. Dunaway. Creating a large topic map by integrating Wandora and Ontopia. Library Hi Tech. 2013(31): 64-75. 